\title{
Redshift stasis of photons at crossing the gravitationally- bound regions revises the supernovae data
}

\begin{abstract}
Zahid Zakir ${ }^{1}$
Abstract

In gravitationally-bound regions (GBR), largest of which are galaxy clusters, metrics is averagely static and here objects, including radiation, do not participate at the cosmological expansion. In the paper the discovery of new observable effects for radiation crossed such regions - the redshift stasis and flux amplification - is reported. The stasis of wavelength appears as a blueshift with respect to a normally expanded flux and the stasis of distances between photons appears as a higher luminosity. Due to smaller inter-cluster distances at earlier epochs, the corrections to observing redshifts and magnitudes grow with distance. It is shown that the influence to the crossing radiation of switching off on expansion flow is significant and it may exceed up to two or three orders the ordinary gravitational effects of clusters. This sufficiently revises the estimations of characteristics of extragalactic objects and the confrontation with observations of cosmological models becomes correct only at accounting of these stasis effects. The stasis effects sufficiently change temperature of cosmic microwave background and its anisotropy also and lead to new blueshift effects, which particularly may appear in observations as a "dark flow".
\end{abstract}

PACS: 98.80.Es, 97.60.Bw, 98.65.Cw, 98.62.Py, 98.62.Ck, 98.62.Qz, 98.80.Jk, 98.70.Vc

Key words: cosmology, gravitation, galaxy clusters, supernovae, redshift, background radiation

Clusters of galaxies are largest gravitationally-bound regions (GBRs) in the Universe. In general relativity (GR), at once after discovery of the cosmological expansion, it has been established [1-3] that the space-time metric in GBR and in its vicinity is averagely static, and the partial transition to the nonstatic cosmological metric begins outside a certain radius $r_{s}$. Let the cluster's centre of mass is at rest under the cosmic microwave background (CMB). Then any local-inertial frame outside $r_{s}$, also resting under $\mathrm{CMB}$, has non-zero receding velocity from the cluster due to participation in the cosmological expansion. Objects in GBR $\left(r<r_{s}\right)$ do not participate in the expansion and behave as in a usual gravitational field.

In such weak fields and non-relativistic velocities it is enough to use the Newtonian theory, in which $r_{s}$ is a "zero acceleration radius", where the "acceleration" $H_{0}^{2} r_{s}$ of a unit mass related by its receding velocity $v_{H}=H_{0} r$, where $H_{0}=h_{0} \cdot 100$ $\mathrm{km} \cdot \mathrm{s}^{-1} \mathrm{Mps}^{-1}$ is the Hubble constant, is balanced by its gravitational acceleration to the cluster of mass $M$ :

${ }^{1}$ Centre for Theoretical Physics and Astrophyics, Tashkent, Uzbekistan; zahidzakir@theor-phys.org 


$$
H_{0}^{2} r_{s}=\frac{G M}{r_{s}^{2}}, \quad r_{s}=\frac{(G M)^{1 / 3}}{H_{0}^{2 / 3}} .
$$

Beyond GBR $r>r_{s}$ a receding velocity from the cluster obeys a "quasi-Hubble" law $v_{H, s}(r)=H_{s} r$, where $H_{s}(r)$ is a "quasi-Hubble" parameter which is less than $H_{0}$ due to gravitying to the cluster. In this transient region it takes place a partial stasis of the cosmological redshift grooving. Here the gravitational acceleration only partially compensates the "cosmological" one and instead of (1) there is a nonzero difference of these accelerations:

$$
H_{s}^{2} r=H_{0}^{2} r-\frac{G M}{r^{2}}, \quad H_{s}=H_{0} \sqrt{1-\frac{r_{s}^{3}}{r^{3}}}, \quad r>r_{s} .
$$

As we see, outside GRB $H_{s}$ quickly enough tends to $H_{0}$ - at $r=(1.25 ; 1.5 ; 2.0 ; 2.5) r_{s}$ we obtain $H_{s}=(0.70 ; 0.84 ; 0.94 ; 0.97) H_{0}$, i.e. in the interval from $r_{s}$ up to $1.5 r_{s}$ the value $H_{s}$ grows from zero up to $84 \%$ of $H_{0}$.

Let us introduce, for simplicity, $r_{s e}$ as an effective radius of GBR of a cluster, in which the expansion absents exactly, but out of which occurs in the full form. We can take $\Delta l_{g} \simeq 2.5 r_{\text {se }}$ as an effective size of the inter-cluster GRB or the stasis region size. At $h_{0}=0.70$ for masses $M=10^{12} \div 10^{15} M_{\odot}$ the values of $r_{s}$ and $\Delta l_{g}$ are presented in Table 1 . Thus, by taking present epoch's mean inter-cluster distance $\Delta l_{c} \sim 5 \div 15 \mathrm{Mps}$, we see that $\Delta l_{g}$ occupies $10 \div 50 \%$ of $\Delta l_{c}$. For rough estimation we can take it of order $\Delta l_{g} \sim(0.15 \div 0.30) \Delta l_{c}$. Thus, at crossing of radiation through GBR of galaxy clusters the redshift does not grow, the intensity and other characteristics evolve as in a static world and become normal one (as in the expanding world) only after leaving this region.

Table 1. GBR of galaxy clusters (Mpc)

\begin{tabular}{|c|c|c|c|c|}
\hline $\mathrm{M}_{\odot}$ & $10^{12}$ & $10^{13}$ & $10^{14}$ & $10^{15}$ \\
\hline$r_{s}$ & 1.2 & 2.6 & 5.6 & 12.1 \\
\hline$\Delta l_{g}$ & 3.0 & 6.5 & 14.0 & 30.0 \\
\hline
\end{tabular}

are related by the observing redshift $z$ :

$$
\frac{a_{0}}{a_{\bar{z}}}=1+\bar{z}, \quad \frac{\lambda_{r}}{\lambda_{e}}=1+z,
$$

In the homogeneous world these two definitions are equivalent, while at the presence of GBRs $z<\bar{Z}$ and the stasis effect correction $\delta z=\bar{z}-z$ appears as an effective blueshift reducing $\bar{Z}$.

The relative blueshift $\delta z / \bar{Z}=1-z / \bar{Z}$ is averagely equal to the ratio of photon's path $\delta l_{\bar{z}}$ in $N$ GBRs to the total path $l_{\bar{z}}=c\left(\tau_{0}-\tau_{\bar{z}}\right)$, where $\tau$ - is the cosmological proper time. At cosmic expansion a mean mass of clusters changes sufficiently slowly, than mean distance between them, so in the first approximation we can take $\Delta l_{g(i)} \approx \Delta l_{g}$ and $\delta l_{\bar{z}}=\Sigma_{i} \Delta l_{g, i} \approx N \Delta l_{g}=\delta l_{g}$, which gives: 


$$
\frac{\delta \mathrm{z}}{\overline{\mathrm{Z}}}=\frac{\delta l_{\overline{\mathrm{z}}}}{l_{\overline{\mathrm{z}}}} \approx \frac{\delta l_{g}}{l_{\overline{\mathrm{z}}}} \rightarrow \frac{l_{0}}{l_{\overline{\mathrm{z}}}} f_{g} .
$$

Here $f_{g} \equiv \delta l_{g} / l_{0} \approx \Delta l_{g} / \Delta l_{c}$ is the ratio of photon's path in GBR of clusters to the present distance up to the source $l_{0}=a_{0} \chi_{0} \approx a_{0} \chi_{\bar{z}}$. At $z \ll 1$ we have $z \sim\left(1-f_{g}\right) \cdot \bar{z}$ which leads to renormalization of $H_{o b s}$ by dividing to the factor $1-f_{g}$.

In the first model-independent approximation, giving lower limit for $\delta z$, we can take for $l_{\bar{z}}$ a mean-arithmetic of distances up to the source at emitting and receiving of photon $l_{\bar{z}} \approx\left(a_{0} \chi_{0}+a_{\bar{z}} \chi_{\bar{z}}\right) / 2$. Taking into account that $\chi_{0} \simeq \chi_{\bar{z}}$ and using Eqs. (3)(4), then we obtain:

$$
\begin{gathered}
l_{\bar{z}} \approx \frac{l_{0}}{2}\left(1+\frac{a_{\bar{z}}}{a_{0}}\right)=l_{0} \frac{1+\bar{z} / 2}{1+\bar{z}}, \\
\delta z \approx \frac{\bar{z}(1+\bar{z})}{1+\bar{z} / 2} f_{g},
\end{gathered}
$$

which gives:

$$
z=\bar{z}-\delta z=\frac{\bar{z}}{1+\bar{z} / 2}\left[(1-f)+\left(\frac{1}{2}-f\right) \bar{z}\right] .
$$

In any cosmological model for the luminosity distance $d_{0}$ corresponds some value $\bar{Z}$, which allows to find $Z$ from Eq. (7) and to compare the model with observations. But, since $\delta \mathrm{z}$ is a correction to the data, it will be more likely to enter it to the data and the magnitude-redshift data tables represent in terms of $\bar{Z}$ by reversing (7):

$$
\bar{z}=\frac{1}{1-2 f}\left[\sqrt{(1-f)^{2}+(1-3 f) z+z^{2} / 4}-(1-f)+\frac{z}{2}\right] .
$$

Let us consider the consequences of switching out on the expansion at crossing GBRs for the apparent luminosity of radiation. At propagation of photons in homogeneous space a source of absolute luminosity $L$ would have the standard apparent luminosity [5] $\bar{l}=L /\left[4 \pi d_{0}^{2}(1+\bar{z})^{2}\right]$. For radiation crossed GBRs $\bar{l}$ should be expressed through the observing apparent luminosity $l$ as:

$$
\bar{l}=l \cdot \frac{D_{\delta z}}{B_{\delta z}^{2} C_{\delta \varepsilon}^{2}} \approx l \cdot B_{\delta z}^{-4} D_{\delta z} .
$$

Here the magnification of $l$ at redshift stasis is given by the coefficient $B_{\delta z}=(1+\bar{z}) /(1+z)$ and due to relative narrowing of the beam at stasis by $C_{\delta \varepsilon}=1+\delta \varepsilon / \varepsilon$ since outside GBR the unperturbed beam with solid angle $\pi \varepsilon^{2}$ expands additionally. The estimation shows that $C_{\delta \varepsilon} \simeq B_{\delta \varepsilon}$ which simplifies Eq. (9). The coefficient $D_{\delta z}>1$ expresses decreasing of apparent luminosity at absorption and scattering due to the increased distance.

The observational data for sources are tabulated as a relation between $\mathrm{Z}$ and the distance modulus $\mu(z)=m-M$, where $m, M$ are apparent and absolute magnitudes. By adding the stasis effect corrections to $z$ and $m$, we calculate $\bar{z}$ and $\bar{\mu}(\bar{z})=\bar{m}-\bar{M}$ by Eqs. (7)-(9), enter them as table's new columns and compare with $\bar{\mu}_{t h}(\bar{z})$ of the model. 
For Type 1A supernovae (SN 1a), accepted as a cosmological standard candle, the spectroscopically-confirmed data for $\mu_{\text {obs }}(z)$ are available up to $z \sim 1.91$. We use 558 objects of Union 2.1 compilation [4] - from total set of 580 objects 7 we remove as contributing to $\chi^{2}$ larger 6 , and for 15 we use data from [8]. To this set then we add three most distant SN 1a with $z=1.55$ [5], $z=1.71$ [6] and $z=1.91$ [7], and 33 objects of the "pure" set [8]. The distance modulus of this data set for $N=594$ SNs (taken at $M=-19.31)$ can be approximated very well $\left(\chi^{2} / N=0.90\right)$ by a simple empirical formula:

$$
\mu_{\text {obs }}(z)=5 \lg \left(z+\gamma z^{2}\right)+5 \lg \left(c / H_{o b s}\right)
$$

at the values of parameters $H_{o b s}=68,6 \mathrm{~km} \cdot \mathrm{s}^{-1} \mathrm{Mps}^{-1}$ and $\gamma=0.57$ (Fig. 1).

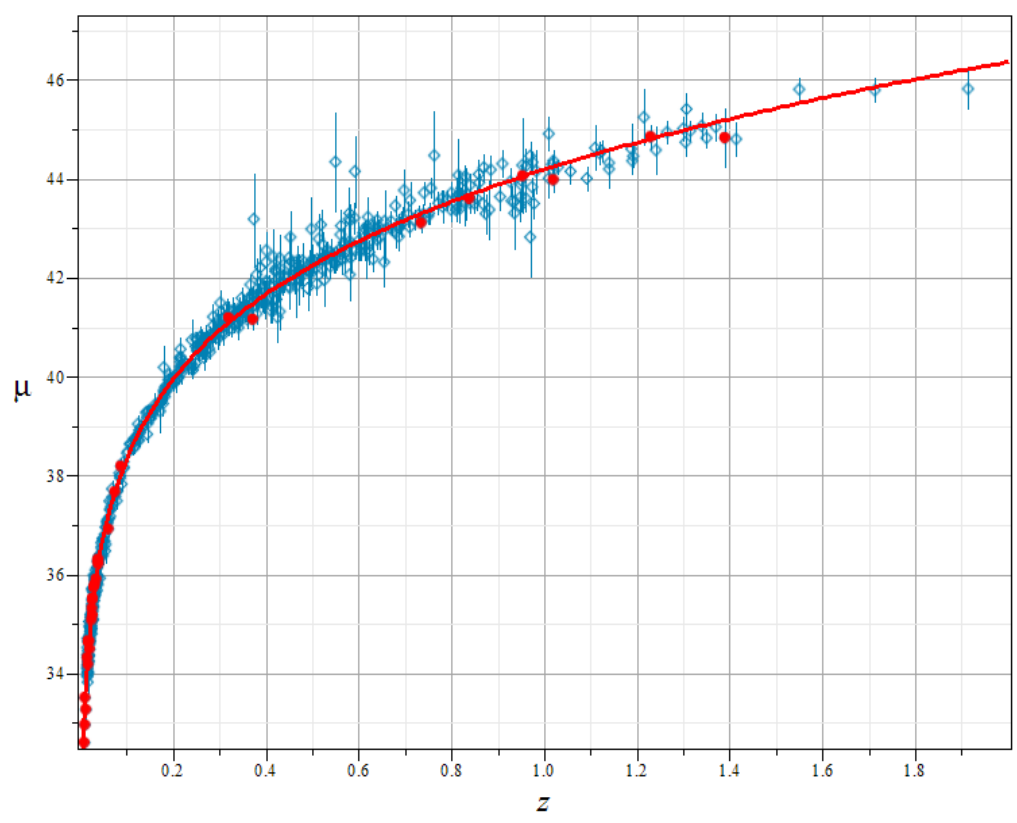

Figure 1. Distance modulus- redshift (z) diagram for SNe Ia for observing $\mu_{o b s}=m-M$ and $z$. Data for 561 objects are taken from [3-7] (blue diamond), circles are set of 33 "pure" SN 1a [8]. The curve corresponds to the empirical formula (10) at $H_{\text {obs }}=68,6 \mathrm{~km} \cdot \mathrm{s}^{-1} \mathrm{Mps}^{-1}$ and $\gamma=0.57$.

At practically lower limit $f_{0}=0.15$ for the fraction of GBR in the mean intercluster distance for the observing $Z$ from (8) we obtain practically lower limit for the stasis corrections $\delta \mathrm{z}$ and corresponding $\bar{z}$ which are presented in Table 2 and Fig. 2.

Table 2. Values of observing $(z)$ and effective $(\bar{z})$ redshifts at $f_{0}=0.15$.

\begin{tabular}{|c|c|c|c|c|c|c|c|c|c|c|}
\hline$z$ & 0.100 & 0.500 & 1.000 & 1.200 & 1.300 & 1.390 & 1.414 & 1.550 & 1.713 & 1.914 \\
\hline$\delta z$ & 0.019 & 0.114 & 0.263 & 0.329 & 0.363 & 0.394 & 0.402 & 0.450 & 0.509 & 0.582 \\
\hline $\bar{Z}$ & 0.119 & 0.614 & 1.263 & 1.529 & 1.663 & 1.784 & 1.816 & 2.000 & 2.222 & 2.496 \\
\hline$\delta \mathrm{z} / \bar{Z}$ & $16,0 \%$ & $18,6 \%$ & $20,8 \%$ & $21,5 \%$ & $21,8 \%$ & $22,1 \%$ & $22,1 \%$ & $22,5 \%$ & $22,9 \%$ & $23,3 \%$ \\
\hline
\end{tabular}

As we see, the blueshifts $\delta z$ in the interval $z=1.0 \div 2.0$ increases by distance almost twice, reaching $20-24 \%$ of $\bar{z}$. At $f_{0}>0.15$ the stasis corrections will increase and become one more sufficient. 
Corrections to absorption and scattering in galos of galaxies are usually of order $\Delta m \approx 0.03$ at $Z=1.0$. By taking into account this estimation and Eq. (9), for preliminary estimations of stasis effect corrections to the true (unperturbed by GBRs) distance modulus we may take the formula:

$$
\bar{\mu}_{o b s}(\bar{z}) \simeq \mu_{o b s}(z)+2 \cdot 5 \lg [(1+\bar{z}) /(1+z)]+(z-\bar{z}) \cdot 0.03 / z .
$$

At last, a curve fitting the corrected distance modulus $\bar{\mu}_{\text {obs }}(\bar{z})$ we also can represent in the form (10), but at slightly different value of parameters only (Fig. 2):

$$
\bar{\mu}_{o b s}(\bar{Z})=5 \lg \left(\bar{Z}+\bar{\gamma} \cdot \bar{Z}^{2}\right)+5 \lg \left(c / \bar{H}_{o b s}\right) .
$$

where $\bar{H}_{\text {obs }}=80,7 \mathrm{~km} \cdot \mathrm{s}^{-1} \mathrm{Mps}^{-1}$ and $\bar{\gamma}=0.69$ at $\chi^{2} / N=0.90$. The relationship:

$$
H_{o b s}=\bar{H}_{o b s}\left(1-f_{0}\right)
$$

gives the previous value $H_{\text {obs }}=68,6 \mathrm{~km} \cdot \mathrm{s}^{-1} \mathrm{Mps}^{-1}$.

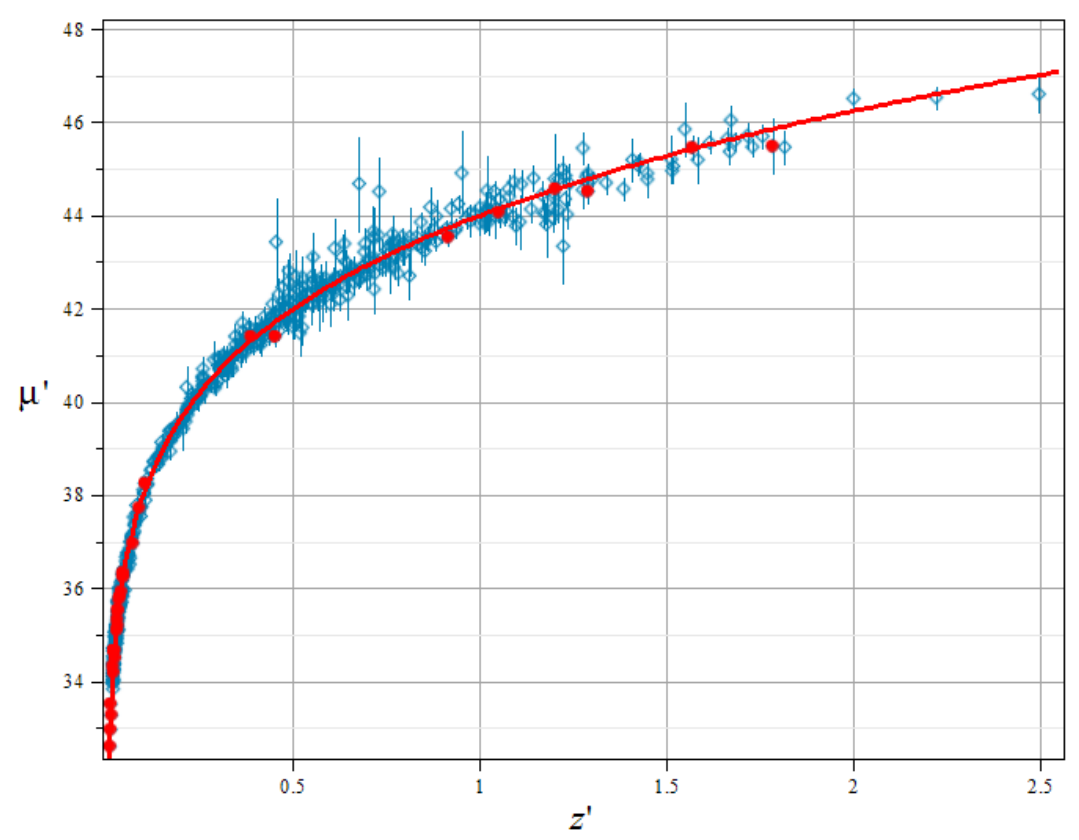

Figure 2. Distance modulus- redshift diagram for $\mathrm{SNe}$ Ia with the stasis effects corrections. The values of $\mu^{\prime} \equiv \bar{\mu}_{o b s}(\bar{Z})=\bar{m}-\bar{M}$ and $Z^{\prime} \equiv \bar{Z}$ are calculated for the objects from Fig.1 by using (8) and (11). The curve corresponds to the empirical formula (12) at $\bar{H}_{o b s}=80,7 \mathrm{~km} \cdot \mathrm{s}^{-1} \mathrm{Mps}^{-1}, \bar{\gamma}=0.69$.

A more consistent approach requires to find the stasis effects corrections to any observing object individually by accounting an influence of any galaxy cluster crossed by the photons. After that the spread of magnitudes and redshifts of objects, probably, becomes sufficiently smaller and the parameters will be defined more precisely.

Thus, the stasis of $z$ and the flux magnification in GBRs of galaxy clusters essentially modify the observational data and they should be taken into account for all extragalactic sources averagely or individually. Distances up to objects remarkably increase, luminosities decrease, thus almost all their characteristics change accordingly.

Let us consider shortly the stasis effects for temperature of the cosmic microwave background (CMB). The observing redshift $z \sim 10^{3}$ for CMB at crossing of large number of inhomogeneities should correspond to higher unperturbed redshift $\bar{Z}>z$ and smaller 
temperature. This means smaller $a_{\bar{z}}$ and higher densities at the recombination epoch, which sufficiently change the standard picture of recombination, including mechanisms of generation of the observing anisotropy.

Main changing of paradigm will be in retreating from the statement about almost sterile propagation of CMB flow after the recombination (with small corrections to known effects) - the temperature stasis at crossing of many GBRs is sufficient and it radically changes the flow. Particularly, there appears a new explanation most part of the observing angular anisotropy as a last time stasis effect in nearest clusters.

As an observational evidence of such mechanism may be the apparent "dark flow" effect [9], which may be in main part a predicting by the present treatment a relative blueshift due to the stasis effect's in GBRs.

More detail study of influences of the stasis effects to CMB, parameters of distant sources and the Universe will be presented in forthcoming papers [10].

\section{References}

1. McVittie G. C. (1933) Mon. Not. R. Astron. Soc. 93, 325.

2. Einstein A., Straus E. G., Rev. Mod. Phys. (1945) 17, 120; (1946) 18, 48.

3. Carrera M. and Giulini D. (2010) Rev. Mod. Phys. 82, 169.

4. Suzuki N. et al. (2012) Astrophys. J. 746, 85.

5. Rodney, S. A., et al. (2012) Astrophys J., 746, 1, 5.

6. Rubin D. et al. (2013) Astrophys J., 763, 1, 35, 10 pp.

7. Jones D. O., et al. (2013) arXiv:1304.0768 [astro-ph.CO].

8. Pruzhinskaya M. V. et al. (2011) Astr. L. 37, 663.

9. Kashlinsky A. et al. (2011) Astrophys. J. 732, 1.

10. Zakir Z. (2013) Theor. Phys., Astrophys.\& Cosmol., 8, 1, p.7; 8, 1, p.15. 